\title{
FUNCTIONAL PEARLS \\ A symmetric set of efficient list operations
}

ROB R. HOOGERWOORD

Department of Mathematics and Computing Science, Eindhoven University of Technology, PO Box 513, $5600 \mathrm{MB}$ Eindhoven, The Netherlands

\section{Introduction}

In this paper we show that it is possible to implement a symmetric set of finite-list operations efficiently; the set is symmetric in the sense that lists can be manipulated at either end. We derive the definitions of these operations from their specifications by calculation. The operations have $\mathrm{O}(1)$ time complexity, provided that we content ourselves with, so-called, amortized efficiency, instead of worst-case efficiency.

The idea behind our design is simple and not new (Gries, 1981), but to be effective its elaboration requires some care. The idea is to represent each list by a pair of lists: a pair $[x, y]$ is used to represent the list $x \# r e v \cdot y$. (Here, a dot denotes functional application.) Thus, each list can be represented in many ways, and it is by judicious exploitation of this freedom that we achieve our goal. We elaborate this in section 3.

\section{Amortized complexity}

Without pretending generality, we introduce the notion of amortized complexity in a form suiting our purpose.

In this section $V$ is a set and $f$ and $t$ are functions of types $V \rightarrow V$ and $V \rightarrow N a t$, respectively. For $v, v \in V$, we interpret $t \cdot v$ as the cost, in some meaning of the word, of evaluation of $f \cdot v$. Now suppose that we are interested in a sequence of successive applications of $f$; i.e. we define a sequence $x$ as follows:

$$
\begin{aligned}
& x_{0} \in V\left(x_{0} \text { is assumed to be known }\right), \text { and } \\
& x_{i+1}=f \cdot x_{i}, \quad 0 \leqslant i .
\end{aligned}
$$

Computation of the first $n+1$ elements of $x$ then costs $\left(\sum i: 0 \leqslant i<n: t \cdot x_{i}\right)$. If the value of this expression, as a function of $n$, is $\mathrm{O}(n)$, then we say that the amortized cost of each of $f$ 's applications (in sequence $x$ ) is $\mathrm{O}(1)$. Of course, this is so if $t$ is $\mathrm{O}(1)$, but this is not necessary: the requirement that $\left(\sum i: 0 \leqslant i<n: t \cdot x_{i}\right)$ is $\mathrm{O}(n)$ is weaker. The introduction of amortized cost reflects our decision to be interested only in the cumulative cost of a sequence of successive operations.

For the sake of simplicity, it would be nice if we could discuss the amortized cost of $f$ without introduction of sequence $x$. This can be done as follows. We introduce 
a function $s$, of type $V \rightarrow N a t$, and we interpret $s \cdot v$ as the amortized cost of evaluation of $f \cdot v$. We try to couple $s$ and $t$ in such a way that, for our sequence $x$, we have:

$$
\left(\Sigma i: 0 \leqslant i<n: t \cdot x_{i}\right) \leqslant\left(\Sigma i: 0 \leqslant i<n: s \cdot x_{i}\right) \text {, for all } n: 0 \leqslant n \text {. }
$$

Consequently, if $s$ is $\mathrm{O}(1)$ then the cumulative cost of computing the first $n+1$ elements of $x$ is indeed $\mathrm{O}(n)$.

The following idea for a suitable coupling is - as far as we know - due to Tarjan (1985). We design, or invent, a function $c$, of type $V \rightarrow N a t$, and define $s$ as follows:

$$
s \cdot v=t \cdot v+c \cdot(f \cdot v)-c \cdot v, \text { for all } v, v \in V .
$$

Under the additional assumption $c \cdot x_{0}=0$, this $s$ satisfies (*).

What does this mean in practice? To prove that a function $f$, with given cost function $t$, has amortized cost $\mathrm{O}(1)$, it suffices to design a natural function $c$, the socalled credit function, satisfying:

$$
\begin{aligned}
& c \cdot x_{0}=0, \text { and } \\
& t \cdot v+c \cdot(f \cdot v)-c \cdot v \quad \text { is, as function of } v, \mathrm{O}(1) .
\end{aligned}
$$

Here $x_{0}$ represents the initial argument - or the initial state - of the computation.

The above remains valid when $f$ represents the elements of a whole class of functions, each having its own cost function $t$. In this case, one and the same credit function must satisfy the above requirement for each pair $f, t$ from this class.

\section{Specifications}

The problem to be solved is to implement an extended set of elementary list operations in such a way that the amortized time complexity of each of these operations is $\mathrm{O}(1)$. For this purpose, the set $L_{*}$ of lists will be represented by a set $V$, say, such that the representation of lists by elements of $V$ is not unique. The abstraction function mapping $V$ to $L_{*}$ is denoted by $\llbracket \cdot \rrbracket$, i.e. $\llbracket s \rrbracket$ is the list represented by $s$, for $s, s \in V$.

We use lists and their associated functions for two purposes, namely to specify the new list operations and to implement them. The functions to be implemented are:

$$
\begin{array}{lll}
\vdash \text { ('left cons') } & \text { and } & - \text { ('rightcons') } \\
\text { lhd('left head') } & \text { and } & r h d(\text { 'right head') } \\
\text { ltl('left tail') } & \text { and } & r t \text { ('right tail'). }
\end{array}
$$

Using $\llbracket \llbracket$ we specify these functions as follows; for any $a$ and for $s, s \in V$ :

$$
\begin{aligned}
\llbracket a \vdash s \rrbracket & =[a]+\llbracket s \rrbracket \\
\llbracket s \dashv a \rrbracket & =\llbracket s \rrbracket+[a] \\
l h d \cdot s & =h d \cdot \llbracket s \rrbracket, \quad \llbracket s \rrbracket \neq[] \\
r h d \cdot s & =h d \cdot(r e v \cdot \llbracket s \rrbracket), \quad \llbracket s \rrbracket \neq[] \\
\llbracket l t l \cdot s \rrbracket & =t l \cdot \llbracket s \rrbracket, \quad \llbracket s \rrbracket \neq[] \\
\llbracket r t l \cdot s \rrbracket & =r e v \cdot(t l \cdot(r e v \cdot \llbracket s \rrbracket)), \quad \llbracket s \rrbracket \neq[] .
\end{aligned}
$$


Remark. From these specifications, the types of these functions can be derived easily.

We also need a representation of the empty list, i.e. we must choose a value []$_{V}$, []$_{v} \in V$, satisfying:

$$
\llbracket[]_{V} \rrbracket=[]
$$

Functions ( $\left.a \vdash^{-}\right)$and $(-\mid a)$, for every $a$, and functions $l t l$ and $r t l$ have type $V \rightarrow V$. They will be implemented in such a way that their amortized time complexity is $\mathrm{O}(1)$. Functions lhd and rhd do not fit into this pattern: they are functions from $V$ to elements. This is no problem: we shall see to it that $l$ h $d$ and $r h d$ have $\mathrm{O}(1)$ (worst-case) time complexity.

\section{Representation}

Our new lists are represented by pairs of old lists, i.e. we choose $V=L_{*} \times L_{*}$. For function $\llbracket \cdot \rrbracket$ we choose:

$$
\llbracket[x, y] \rrbracket=x \quad r e v \cdot y .
$$

This representation leaves us no choice for the definition of []$_{V}$ : the only solution of the equation $x, y:[]=x+r e v \cdot y$ is [], []; hence:

$$
[]_{V}=[[],[]] \text {. }
$$

We now derive a definition for $l$ hd:

$$
\begin{aligned}
& \text { lhd } \cdot[x, y] \\
= & \quad \text { specification of } l h d\} \\
& h d \cdot \llbracket[x, y] \rrbracket \\
= & \{\text { definition of } \llbracket \cdot \rrbracket\} \\
& h d \cdot(x+r e v \cdot y) \\
= & \{\text { definitions of and } h d\} \\
& \text { if } x \neq[] \rightarrow h d \cdot x \\
& \square x=[] \rightarrow h d \cdot(r e v \cdot y)
\end{aligned}
$$

fi.

Evaluation of $h d \cdot(r e v \cdot y)$ takes $\mathrm{O}(\# y)$ time; hence, the definition thus obtained does not have $\mathrm{O}(1)$ time complexity. It does, however, have $\mathrm{O}(1)$ time complexity in the special case $x \neq[] \vee \# y \leqslant 1$, or equivalently, $1 \leqslant \# x \vee \# y \leqslant 1$. Therefore, we

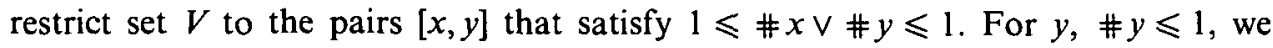
have $r e v \cdot y=y$; thus, we obtain the following definition for lhd:

$$
\begin{array}{r}
\operatorname{lhd} \cdot[x, y]=\text { if } x \neq[] \rightarrow h d \cdot x \\
0 x=[] \rightarrow h d \cdot y
\end{array}
$$

fi.

By symmetry, we restrict set $V$ to those pairs $[x, y]$ satisfying $1 \leqslant \# y \vee \# x \leqslant 1$ as 
well. Together, these two restrictions define set $V$ as a subset of $L_{*} \times L_{*}$. The relation defining this subset, also called the representation invariant, is $Q$, with:

$$
Q: \quad(1 \leqslant \# x \vee \# y \leqslant 1) \wedge(1 \leqslant \# y \vee \# x \leqslant 1) .
$$

The definition for rhd then becomes (notice the symmetry):

$$
\begin{array}{r}
r h d \cdot[y, x]=\text { if } x \neq[] \rightarrow h d \cdot x \\
\| x=[] \rightarrow h d \cdot y
\end{array}
$$

fi.

\section{Left and right cons}

The derivation of definitions for $t$ and $\dashv$ is straightforward if we temporarily forget the proof obligation with respect to $Q$. We perform these derivations in parallel:

$$
\begin{aligned}
& \llbracket a \vdash[x, y] \rrbracket \\
& =\quad \text { specification of } \vdash \text { \} } \\
& {[a]+\llbracket[x, y] \rrbracket} \\
& =\quad \text { definition of } \llbracket \cdot \rrbracket\} \\
& {[a]+x+\mathrm{rev} \cdot y} \\
& =\text { \{list calculus }\} \\
& (a: x)+r e v \cdot y \\
& =\quad \text { definition of } \llbracket \cdot \rrbracket\} \\
& \llbracket[a: x, y] \rrbracket \text {. } \\
& \llbracket[y, x] \dashv a \rrbracket \\
& =\{\text { specification of }-1\} \\
& \llbracket[y, x] \rrbracket+[a] \\
& =\quad \text { definition of } \llbracket \cdot \mathbb{1}\} \\
& y+r e v \cdot x+[a] \\
& =\{\text { list calculus }\} \\
& y+\operatorname{rev} \cdot(a: x) \\
& =\{\text { definition of } \llbracket \cdot \mathbb{} \text { \} } \\
& \llbracket[y, a: x] \rrbracket \text {. }
\end{aligned}
$$

Thus, we conclude that the specifications of $\vdash$ and $\dashv$ are satisfied by:

$$
\begin{aligned}
a \vdash[x, y] & =[a: x, y] . \\
{[y, x] \vdash a } & =[y, a: x] .
\end{aligned}
$$

The expression $[a: x, y]$ will only satisfy $Q$ if $1 \leqslant \# y$. For the special case $y=[]$, we redo the above derivation:

$$
\begin{aligned}
& \llbracket a \vdash[x,[]] \rrbracket \\
= & \{\text { as before, with }[] \text { for } y\} \\
& {[a]+x \# \text { rev } \cdot[] } \\
= & \{\text { rev } \cdot[]=[],[] \text { is the identity of } \#\} \\
& {[a] \# x } \\
= & \{[x,[]] \text { satisfies } Q, \text { hence } \# x \leqslant 1, \text { hence } x=r e v \cdot x\} \\
& {[a]+\text { rev } \cdot x } \\
= & \{\text { definition of } \llbracket \cdot \rrbracket\} \\
& \llbracket[[a], x] \rrbracket .
\end{aligned}
$$


Expression $[[a], x]$ satisfies $Q$. Thus, we obtain the following definition for $\vdash$ and, similarly, for $\dashv$ :

$$
\begin{aligned}
a \vdash[x, y]= & \text { if } y \neq[] \rightarrow[a: x, y] \\
& \forall y=[] \rightarrow[[a], x] \\
& \text { fi, } \\
{[y, x] \dashv a=} & \text { if } y \neq[] \rightarrow[y, a: x] \\
& \text { \& } y=[] \rightarrow[x,[a]]
\end{aligned}
$$

fi.

The (normal) time complexity of these definitions is $O(1)$. In order that their amortized time complexity is $\mathrm{O}(1)$ as well, the credit function $c$ must be chosen such that its value increases by a bounded amount under these operations; that is, $c \cdot(a \vdash[x, y])-c \cdot[x, y]$ must be bounded from above by a constant.

\section{Left and right tail}

We now derive definitions for $l t l$ and $r t l$. These derivations do not yield efficient definitions, but they do provide information on how the credit function can be chosen such that these definitions have $O(1)$ amortized time complexity.

For $l t l \cdot[x, y]$, with precondition $\llbracket[x, y] \rrbracket \neq[]$, we derive:

$$
\begin{aligned}
& \llbracket l t l \cdot[x, y] \rrbracket \\
= & \{\text { specification of } l t l\} \\
& t l \cdot \llbracket[x, y] \rrbracket \\
= & \quad\{\text { definition of } \llbracket \cdot \rrbracket\} \\
& t l \cdot(x+r e v \cdot y) .
\end{aligned}
$$

Further manipulation of this formula requires distinction of the cases $x \neq[]$ and $x=[]$.

For the case $x=[]$ we have:

$$
\begin{aligned}
& t l \cdot(x+r e v \cdot y) \\
& =\{x=[]\} \\
& t l \cdot(\mathrm{rev} \cdot \mathrm{y}) \\
& =\{Q \wedge \llbracket[x, y] \rrbracket \neq[] \wedge x=[] \Rightarrow \# y=1 \text {, hence } t l \cdot(r e v \cdot y)=[]\} \\
& \text { [] } \\
& \left.=\quad \text { specification of }[]_{V}\right\} \\
& \llbracket[]_{v} \rrbracket \\
& \left.=\quad \text { definition of }[]_{\mathrm{v}}\right\}
\end{aligned}
$$

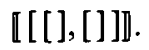

Hence, for the case $x=[]$ we must choose - no freedom $-l t l \cdot[x, y]=[[],[]]$. 
For the case $x \neq[]$ we derive:

$$
\begin{aligned}
& t l \cdot(x \quad r e v \cdot y) \\
= & \{x \neq[], \text { definitions of } \# \text { and } t\} \\
& t l \cdot x \# \text { rev } \cdot y \\
= & \{\text { definition of } \llbracket \cdot \rrbracket\} \\
& \llbracket[t l \cdot x, y] \rrbracket .
\end{aligned}
$$

So, for the case $x \neq[]$, we may choose $l t l \cdot[x, y]=[t l \cdot x, y]$, provided that this expression satisfies $Q$, i.e. we must prove $Q \Rightarrow Q(x, y \leftarrow t l \cdot x, y)$, where $\leftarrow$ denotes substitution. Assuming $Q$ we derive:

$$
\begin{aligned}
& Q(x, y \leftarrow t l \cdot x, y) \\
= & \{\text { definition of } Q\} \\
& (1 \leqslant \#(t l \cdot x) \vee \# y \leqslant 1) \wedge(1 \leqslant \# y \vee \#(t l \cdot x) \leqslant 1) \\
= & \{\text { definitions of } t l \text { and } \#\} \\
& (2 \leqslant \# x \vee \# y \leqslant 1) \wedge(1 \leqslant \# y \vee \# x \leqslant 2) \\
= & \{Q \Rightarrow 1 \leqslant \# y \vee \# x \leqslant 2\} \\
& 2 \leqslant \# x \vee \# y \leqslant 1 \\
\leftarrow & \{\text { predicate calculus }\} \\
& 2 \leqslant \# x .
\end{aligned}
$$

So, for the special case that $x$ has at least two elements, the above definition for $l t l$ is correct. The remaining case is that $x$ is a singleton list:

$$
\begin{aligned}
& t l \cdot x+r e v \cdot y \\
= & \{\# x=1\} \\
& {[]+r e v \cdot y } \\
= & \{[] \text { is the identity of } \#\} \\
& r e v \cdot y \\
= & \{\text { introduce } u \text { and } v \text { such that } y=u+v \quad \text { (note } 1, \text { see below) }\} \\
& r e v \cdot(u+v) \\
= & \{\text { list calculus }\} \\
& r e v \cdot v+r e v \cdot u \\
= & \{\text { definition of } \llbracket \cdot \rrbracket\} \\
& \llbracket[\text { rev } \cdot v, u] \rrbracket .
\end{aligned}
$$

So, for the case $\# x=1$ we may choose $l t l \cdot[x, y]=[\operatorname{rev} \cdot v, u]$, where $u+v=y$.

Note 1: The decision to split $y$ into parts $u$ and $v$ is inspired by the desire to transform $r e v \cdot y$ into a pair of values. By not further specifying $u$ and $v$ we retain the freedom to choose the most efficient representation. 
Evaluation of $[r e v \cdot v, u]$ takes $\mathrm{O}(\# y)$ time, independently of how $u$ and $v$ have been chosen. To obtain $\mathrm{O}(1)$ amortized time complexity, the credit function $c$ must satisfy:

$$
t \cdot y+c \cdot[r e v \cdot v, u]-c \cdot[x, y] \leqslant K,
$$

where $t \cdot y$ denotes the time needed to evaluate $[\mathrm{rev} \cdot v, u]$ and for some constant $K$. Because $t \cdot y$ is $\mathrm{O}(\# y)$ we may safely assume that $t \cdot y \leqslant \# y+1$; then we have:

$$
\begin{aligned}
& t \cdot y+c \cdot[r e v \cdot v, u]-c \cdot[x, y] \leqslant K \\
\equiv \quad & \{\text { calculus }\} \\
& c \cdot[x, y]-c \cdot[\text { rev } \cdot v, u] \geqslant t \cdot y-K \\
\Leftarrow \quad & \{t \cdot y \leqslant \# y+1, \text { transitivity of } \leqslant\} \\
& c \cdot[x, y]-c \cdot[\text { rev } \cdot v, u] \geqslant \# y+1-K .
\end{aligned}
$$

\section{The credit function}

So as not to destroy the symmetry we require $c$ to be symmetric in $x$ and $y$; so, $c \cdot[x, y]=c \cdot[y, x]$, for all $x$ and $y$. One of the simplest such functions is given by:

$$
c \cdot[x, y]=\# x+\# y .
$$

By a simple calculation it can be shown that this definition is equivalent to:

$$
c \cdot[x, y]=\# \llbracket[x, y] \rrbracket .
$$

This function is not useful, for two reasons. First, the length of the represented list increases or decreases by just 1 under each of the list operations. So, amortized and normal complexity coincide. Phrased differentially, the idea of amortized complexity amounts to choosing a function $c$ that allows, every now and then, more substantial decreases of its value. Second, the second definition shows that $c$ is invariant under changes of representation; so, this $c$ gives no heuristic guidance when we exploit the freedom to apply changes of representation.

A function $c$ that does satisfy these requirements is:

$$
c \cdot[x, y]=|\# x-\# y| \text {. }
$$

We leave the proof that the value of $c$ increases by at most 1 under the left and right cons operations as an exercise to the reader.

We now use this $c$ to complete the design of the definitions for $l t l$ and $r t l$. In the previous section we have derived that, for the special case $\# x=1$, values $u$ and $v$ must be chosen such that $c \cdot[x, y]-c \cdot[r e v \cdot v, u] \geqslant \# y+1-K$, for some constant $K$. We have:

$$
\begin{aligned}
& c \cdot[x, y] \\
= & \{\text { definition of } c\} \\
& |\# x-\# y| \\
= & \{\# x=1\} \\
& |\# y-1| \\
\geqslant & \{\text { definition of }|\cdot|\} \\
& \# y-1 .
\end{aligned}
$$


Furthermore, we must see to it that $c \cdot[\operatorname{rev} \cdot v, u]$ is not too large:

$$
\begin{aligned}
& c \cdot[\text { rev } \cdot v, u] \\
= & \quad\{\text { definition of } c\} \\
& |\#(r e v \cdot v)-\# u| \\
= & \quad\{\#(r e v \cdot v)=\# v\} \\
& |\# v-\# u| .
\end{aligned}
$$

By choosing the lengths of $u$ and $v$ as equal as possible we achieve $c \cdot[\operatorname{rev} \cdot v, u] \leqslant 1$. So, we have:

$$
c \cdot[x, y]-c \cdot[r e v \cdot v, u] \geqslant \# y-2
$$

Therefore, we choose $u$ and $v$ such that:

$$
u+v=y \wedge \# u \leqslant \# v \leqslant \# u+1 .
$$

The pair $[r e v \cdot v, u]$ thus specified satisfies $Q$; this follows from a simple calculation. From this specification it also follows that $\# u=\# y$ div 2 . For any $k, 0 \leqslant k \leqslant \# y$, the equation $u, v: u+v=y \wedge \# u=k$ has exactly one solution which we denote by $y \uparrow k, y \downarrow k$. This solution can be computed, in $\mathrm{O}(k)$ time. Therefore, we define $u$ and $v$ by $u=y \uparrow k$ and $v=y \downarrow k$, with $k=\# y$ div 2 .

Putting all the pieces together we obtain the following definitions for $l t l$ and its symmetric counterpart $r t l$ :

$$
\begin{aligned}
& l t l \cdot[x, y]=\text { if } \# x=0 \rightarrow[[],[]]
\end{aligned}
$$

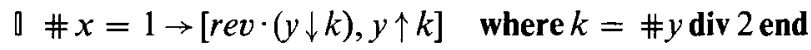

$$
\begin{aligned}
& \text { ॥ } \# x \geqslant 2 \rightarrow[t l \cdot x, y] \\
& \text { fi. } \\
& r t l \cdot[y, x]=\text { if } \# x=0 \rightarrow[[],[]] \\
& \text { \# } x=1 \rightarrow[y \uparrow k, r e v \cdot(y \downarrow k)] \quad \text { where } k=\# y \operatorname{div} 2 \text { end } \\
& \text { o \# } x \geqslant 2 \rightarrow[y, t l \cdot x]
\end{aligned}
$$

fi.

\section{Epilogue}

A formalized notion of amortized complexity turns out to be of heuristic value for the derivation of efficient programs. In our example, we have chosen function $c$ with no more justification than an appeal to a few general criteria. Once $c$ has been chosen, the definitions for $l t l$ and $r t l$ can be completed in a rather straightforward way.

In terms of the list representation used in this paper, reversal of a list is a trivial operation: we have $r e v \cdot \llbracket[x, y] \rrbracket=\llbracket[y, x] \rrbracket$, for all $x$ and $y$. Hence, rev can be implemented efficiently by a function $\operatorname{Rev}$, defined by $\operatorname{Rev} \cdot[x, y]=[y, x]$. Thus, list reversal becomes an $\mathrm{O}(1)$ operation. 


\section{Acknowledgements}

To Berry Schoenmakers for drawing my attention to the above definition of $R e v$, and to David Gries for his comments on an earlier version of this paper.

\section{References}

Gries, D. 1981. The Science of Programming. Springer-Verlag.

Tarjan, R. E. 1985. Amortized computational complexity. SIAM J. on Algebraic and Discrete Methods, 6: 306-318. 